\title{
INVESTIGACIÓN/RESEARCH
}

Recibido: 24/05/2016 --- Aceptado: 02/07/2016 --- Publicado: 15/07/2016

\section{COMUNICACIÓN DE LA CIENCIA Y LA TECNOLOGÍA EN LAS UNIVERSIDADES ECUATORIANAS: ESTUDIO PRELIMINAR DEL IMPACTO Y PERCEPCIÓN ENTRE LA POBLACIÓN UNIVERSITARIA}

Sofía Cabrera: Universidad de las Américas. Ecuador

Emma Camarero: Universidad Loyola Andalucía. España.

ecamarero@uloyola.es

\section{RESUMEN:}

Esta investigación es un estudio preliminar y una contextualización de la divulgación de la ciencia y la tecnología a través de las redes sociales en las universidades ecuatorianas y configura los primeros resultados de la tesis doctoral de la principal autora. En concreto este estudio se centra en las instituciones de la provincia de Pichincha. En febrero de 2016 se desarrolló una encuesta en línea, con una muestra de 50 estudiantes por cada una de las universidades seleccionadas, para definir un estado de la cuestión que sirva de base para identificar y valorar los medios digitales universitarios más usados para informarse por parte de la población estudiantil. Una vez realizada esa encuesta, se procedió al análisis de las publicaciones de Facebook, la red social más utilizada por los estudiantes en dicho período. El objetivo es identificar los modelos de divulgación de la ciencia que utilizan las universidades para seleccionar prácticas de comunicación efectivas en medios digitales.

PALABRAS CLAVE: universidad - ciencia - tecnología - divulgación - redes sociales

\footnotetext{
${ }^{1}$ Nombre y Apellidos del autor de contacto: Doctoranda en Lógica y Filosofía de la Ciencia de la Universidad de Salamanca. Máster en Estudios Sociales de la Ciencia y Tecnología. Comunicadora Social de la Universidad Central del Ecuador. Docente Universitaria.
}

Correo:sofia_sice@usal.es 


\title{
COMMUNICATION OF SCIENCE AND TECHNOLOGY IN UNIVERSITIES OF ECUADOR: PRELIMINARY STUDY OF IMPACT AND PERCEPTION IN THE UNIVERSITY POPULATION
}

\begin{abstract}
:
This research is a preliminary study and contextualization of the popularization of science and technology through social networks in Ecuadorian universities and set up the first results of the doctoral thesis lead author. Specifically, this study focuses on the institutions of Pichincha-Ecuador. In February 2016, an online survey was developed, with a sample of 50 students of each of the selected universities to define an initial statement to identify and assess the most widely college digital media use for information by of the student population. Once that survey, we proceeded to the analysis of the publications of Facebook, the social network most used by the students in that period. The purpose is to identify models of popularizing science used by universities to select effective communication practices in digital media.
\end{abstract}

KEY WORDS: university - science - technology -media literacy - contextualization network.

\section{INTRODUCCIÓN}

Cuando se utilizan los términos de comunicación o divulgación de la ciencia es necesario contextualizarlo al lugar que se hace referencia, ya que aunque el uso de sus palabras puede cambiar, el significado de transmisión pública y democrática del conocimiento se mantiene. Los términos más empleados en Iberoamérica son: Apropiación social de la ciencia (Colombia), Chile y Argentina (Apropiación social del conocimiento científico), España, México y otros países latinoamericanos (divulgación de la ciencia) (Lozano, 2003). Todos estos términos afianzan a la divulgación dentro de un marco comunicacional que busca la ampliación y actualización del conocimiento científico con dos condiciones: que sean tareas hechas fuera de la enseñanza académica formal sin el objetivo de formar especialistas ni de perfeccionarlos en su propia en su área de conocimiento (Erazo, 2007).

En el caso de Ecuador se trabaja con el término "divulgación" de acuerdo a la influencia generada por todo Iberoamérica. En el país son pocas y hasta limitadas las experiencias de divulgación de la ciencia que se han desarrollado durante los últimos treinta años. La transformación constante del Estado en la construcción de políticas públicas que sustenten a la ciencia como pilar fundamental de su desarrollo social, fue el principal problema para la generación de productos y espacios que popularicen una cultura científica sólida entre la sociedad en general. 
La falta de una política científica orientada al estudio y a la solución de problemas nacionales, así como al fomento de una conciencia cívica, ha conllevado a varios países de Iberoamérica a un estado de extrema dependencia en los aspectos económico, industrial, político, científico y tecnológico. De esta manera, la ciencia incumple con su cometido social, porque no contribuye al desarrollo adecuado de los pueblos. (Erazo, 2007:118)

En los últimos treinta años el Ecuador ha pasado por varios cambios en la consolidación de un organismo que coordine las acciones de ciencia y tecnología, pasando de una Fundación que dependía de la Vicepresidencia y administraba fondos para proyectos de investigación, a una Secretaría Nacional con rango de ministerio encargada de las políticas de educación superior, ciencia, tecnología e innovación. (Salazar, 2015)

Los cambios generados han redefinido el tratamiento de la ciencia desde un sistema neoliberal que la consideraba una quimera, es decir un sueño que no se materializa en acciones prácticas, ajustada a iniciativas privadas sin respaldo presupuestario estatal, hasta el desarrollo de un nuevo modelo que prioriza la transformación de la matriz productiva basada en un Sistema de Economía Social del Conocimiento. Sin embargo, en este constante cambio de planeación y diseño de políticas públicas, la divulgación científica no ha sido abordada con la relevancia que requiere para diseñar líneas de acción determinadas en la socialización de la producción científica del país.

A pesar que en los años setenta se incluyen leyes y decretos como políticas públicas de CyT, no fueron suficientes para crear un verdadero Sistema Científico-Tecnológico que se compare a los desarrollados en otros países de América Latina en la misma época. En los años noventa las condiciones para la ciencia y tecnología en el país tampoco mejoraron sustancialmente, debido a la falta de un presupuesto específico destinado a las actividades de Investigación y Desarrollo (I+D). La actividad de las universidades continúa débil, los centros gubernamentales de investigación desarticulados entre sí y no existe el desarrollo de una industria innovativa (Salazar, 2015).

En la última década, el gobierno de Rafael Correa ha sido el promotor del cambio de sistema de economía social del conocimiento. Si se realiza una comparación con los años previos al punto de inicio del gobierno (2007) con el proceso que se ha generado hasta la actualidad, se observan una serie de intentos por legislar a la ciencia, tecnología e innovación, pero con escasos instrumentos operacionales de política pública, sin una orientación determinada que defina las líneas de investigación para proyectos y largos intervalos de tiempo entre decisión y ejecución.Es decir, un proceso demasiado burocrático y con un marcado desinterés por parte de los diferentes gobiernos para lograr la construcción de un sistema eficaz de desarrollo de la ciencia, tecnología e innovación en Ecuador. (Salazar, 2015) 
En junio de 2011 se empieza la redacción de un Plan Nacional de Ciencia, Tecnología, Innovación y Saberes, documento que busca convertirse en una guía con estrategias y lineamientos que resguarden el accionar de la CyT en el país. Aunque la construcción de este Plan se realizó bajo un modelo participativo que buscaba la interacción de diferentes actores de la sociedad civil: universidades, institutos de investigación, empresas públicas y privadas, etc., mediante la ejecución talleres a lo largo de todo el país, tuvo la misma suerte que las ediciones de planes anteriores al gobierno de Rafael Correa, no se convirtió en documento oficial, pero permitió el desarrollo de políticas que actualmente ha sido agrupadas dentro de una ley que se ha denominado como "Código de Economía Social de los Conocimientos, la Creatividad y la Innovación"CÓDIGO INGENIOS 2 .

El Código Ingenios se define como un documento oficial que normará el Sistema de Ciencia y Tecnología en Ecuador. Sin embargo, hasta la edición de este artículo se encuentra en el segundo debate para su aprobación en la Asamblea Nacional por lo que aun no ha entrado en vigencia. En esta ley se incluye por primera vez un apartado relacionado con la comunicación pública:

Artículo 117.- Comunicación pública.- Se entiende por comunicación pública todo acto por el cual una pluralidad de personas, reunidas o no en un mismo lugar, y en el momento en que individualmente decidan, pueda tener acceso a la obra sin previa distribución de ejemplares a cada una de ellas. En especial, se encuentran comprendidos los siguientes actos:

1.- Representaciones escénicas mediante cualquier medio o procedimiento.

2.- Proyección pública de obras audiovisuales

3.- Emisión de obras de difusión mediática (radio, prensa, televisión) para la difusión inalámbrica

4.- Transmisión y retransmisión de cable, fibra óptica y otro procedimiento similar

5.- Exposición pública de obras de arte o sus reproducciones;

6.- Difusión pública, por cualquier procedimiento conocido o por conocerse, de palabras, signos, sonidos o imágenes y que exceda del ámbito privado. (Sección IV, Contenidos de Derechos de Autor: COESC, 2015)

Como se presenta previamente, la comunicación pública relacionada con la ciencia y tecnología empieza a legislarse por primera vez dentro de un código que busca gestionar el Sistema de Ciencia y Tecnología en Ecuador. Sin embargo, aun se consideran actividades generales que se estipulan dentro de los derechos de autor, y no establecen normativas específicas para todos los actores que interactúan en el sistema.

\footnotetext{
${ }^{2}$ Acceso al portal oficial de CÓDIGO INGENIOS http://goo.gl/9H86Pk
} 
Por todo lo anteriormente dicho, el estudio que se presenta en este artículo es parte de una tesis doctoral que pretende analizar el manejo, impacto y percepción de las estrategias de comunicación pública de ciencia y tecnología en las universidades ecuatorianas, tiene como objetivo primordial consolidar un plan de buenas prácticas de divulgación universitaria, y convertirse en una propuesta que podría alimentar el artículo 117 de la política del Código INGENIO; en donde se definan las funciones de la comunicación pública de la ciencia que cumplen los actores universitarios en el Sistema, campo que aun no ha sido determinado completamente. Además, esta investigación adquiere alta relevancia como aporte analítico para las universidades en su proceso de optimización de canales de comunicación en pro de la divulgación de su cultura científica.

De manera puntual, este estudio preliminar pretende analizar por qué es importante para las universidades invertir en procesos de comunicación efectivos que les permitan divulgarla producción científica que generan y fortalecen la construcción de una cultura científica, y así consolidar su reconocimiento social. Para contextualizar el concepto de cultura científica resulta interesante utilizar la definición de Leonardo Vaccarezza:

Se entiende la cultura científica como comprensión de la dinámica social de la ciencia, de manera que se tejen, en una interrelación entre productores de conocimientos científicos y otros grupos sociales, todos ellos como partícipes del devenir de la cultura, produciendo significados cuyos origenes y justificaciones provienen desde distintas prácticas, intereses, códigos normativos y relaciones de poder, entendiéndose como un devenir continuo. (Vaccarezza, 2008:110)

Como explica Vaccarezza, la cultura científica es la comprensión de la dinámica social de la ciencia y la interacción que ésta teje con diferentes actores, no especialistas, para la construcción de significados comunes sobre el tema científico. Por este motivo es interesante analizar el caso de las universidades ecuatorianas en la construcción de esta cultura desde las diferentes estrategias de comunicación que utilizan diariamente.

En este estudio preliminar se hace un análisis de cuáles son los principales medios digitales universitarios que utilizan los estudiantes para informarse; posteriormente a esos resultados se analiza la red social Facebook, al ser considerada por los estudiantes como la principal fuente de información digital universitaria. El estudio busca analizar el impacto de las publicaciones de esta plataforma social sobre temas de ciencia y tecnología y presentar datos que permitan a los encargados del manejo de estas redes sociales optimizar las estrategias de difusión basadas en el contenido, lenguaje y públicos objetivos.

\section{2.- OBJETIVOS}

Los objetivos que motivan esta investigación preliminar están relacionados con 
describirla interacción de los estudiantes con los medios digitales universitarios y la dinámica de publicación de los fan pages de facebook de las universidades como plataformas de divulgación de la cultura científica, para realizar un estado de la cuestión sobre el manejo de canales de comunicación en las universidades y resaltar las acciones positivas sobre su manejo y uso que pueden ser incluidas dentro de un modelo de plan universitario de divulgación científica:

a) Interacción de los estudiantes con las redes sociales universitarias, su percepción y aceptación como medios de divulgación científica.

b) Manejo de la Red social facebook como plataforma comunicacional efectiva de divulgación científica universitaria.

El papel de las universidades en la divulgación científica es fundamental, debido a que, además de la docencia y la investigación, la transferencia del conocimiento a la sociedad en general es una de sus misiones, por lo que se requiere la constante actualización de canales de comunicación pública, para cumplir con este cometido. (Lascurain, M. y Sanz, Elías, 2009)

\section{3.- METODOLOGÍA}

Como se explicó previamente, este estudio es parte de una tesis doctoral que ha tomado como muestra seis universidades de la Provincia de Pichincha en Ecuador. Este apartado preliminar contiene el análisis de dos objetos de estudio específicos: los estudiantes universitarios y la red social facebook. Para conocer la percepción de los estudiantes sobre los medios digitales de las universidades seleccionadas se aplicó una encuesta en línea a una muestra intencional y al mismo tiempo se analizó las publicaciones de ciencia y tecnología de los fan page oficiales de las universidades del mes de febrero 2016.

El estudio de Brito, J. G. Laaser, W. Toloza, E. A. (2012) sobre El uso de redes sociales por parte de las universidades a nivel institucional utiliza esta metodología de análisis intencional y comparativo de cinco universidades iberoamericanas para investigar el uso/implementación de redes sociales por parte de las universidades, debido a la importancia que tienen estos nuevos medios en la gestión de la comunicación institucional entre y dentro de varios grupos de actores. Los autores denominan a este modelo de estudio como "communication analytics", en donde utilizan encuestas, entrevistas y análisis de los fan pages oficiales de las instituciones, discutiendo cifras y métodos estadísticos disponibles, para analizar el impacto de datos brindados en redes sociales. 


\subsection{Criterios de Selección tamaño de la muestra de universidades en la región de Pichincha}

Como se manifestó previamente, en la última década el Ecuador ha desarrollado grandes cambios en las áreas de educación, ciencia y tecnología, con el objetivo de fortalecer la producción del conocimiento, como generador de un cambio de matriz productiva y fortalecer un Sistema de Ciencia y Tecnología basado en la Economía Social del Conocimiento. Este cambio es realizado desde una visión política, encaminado por el gobierno de Rafael Correa, quien asume la presidencia desde el 15 de enero de 2007 hasta la actualidad, y busca dar un giro a los procesos educativos para fortalecer la excelencia académica medida con estándares de calidad internacional.

Una de las acciones generadas en este proceso es la creación de un Consejo de Evaluación, Acreditación y Aseguramiento de la Calidad de la Educación Superior (CEAACES), encargado de dirigir un proceso para categorizar a las instituciones de educación superior en Ecuador, tomando en cuenta el análisis de parámetros como: academia, eficiencia académica, investigación, organización e infraestructura. Esta clasificación se divide de $A-E$, considerando la primera como excelencia académica y la última como deficiencia académica, lo que obligó el cierre definitivo de las instituciones.

De acuerdo a este sistema de categorización, en esta investigación se consideran solo universidades de las categorías A-B, según el último informe presentado por el CEAACES $^{3}$ el 10 de mayo de 2016.

Las seis universidades seleccionadas se detallan en el siguiente cuadro, de acuerdo a la categoría en la que se encuentran actualmente.

\begin{tabular}{|c|l|l|}
\hline CATEGORÍA & \multicolumn{1}{|c|}{ PÚBLICA } & \multicolumn{1}{|c|}{ PRIVADA } \\
\hline \multirow{5}{*}{ A } & -Escuela Politécnica Nacional -EPN & \\
& (9.014 estudiantes) & -Universidad San Francisco de \\
& -Universidad de las Fuerzas & $\begin{array}{l}\text { Quito-USFQ } \\
(6.000 \text { estudiantes })\end{array}$ \\
& Armadas - ESPE & \\
& (6.229 estudiantes) & -Pontificia Universidad Católica del \\
& & Ecuador -PUCE \\
& Universidad Central del Ecuador- & (9.369 estudiantes) \\
B & UCE & -Universidad de las Américas - \\
& (38.671 estudiantes) & UDLA (13.932) \\
\hline
\end{tabular}

TABLA 1: Categorización universidades

Fuente: Elaboración propia (2016) basado en datos oficiales de cada institución

\footnotetext{
${ }^{3}$ Informe CEAACES- Mayo 2016 http://goo.gl/T544gI
} 
Como se observa en el cuadro presentado, las universidades seleccionadas cuentan con una cifra significativa de población estudiantil, lo cual ratifica su posicionamiento social en la provincia; aunque tienen evidentes diferencias por su carácter público o privado, y sus líneas de acción académica, cuentan con características como el nivel de aceptación social que las convierten en similares. Las instituciones seleccionadas se encuentran ubicadas en la provincia de Pichincha, cuatro en Quito y dos en los valles aledaños a la ciudad.

\subsection{Encuesta de opinión a estudiantes universitarios}

La primera herramienta que se desarrolla en esta fase preliminares la aplicación de una encuesta de opinión a una muestra intencional de estudiantes (50 por cada universidad seleccionada), mediante el software Survey monkey, para conocer su percepción y aceptación sobre los medios digitales universitarios como plataformas de divulgación de la cultura científica.

Esta encuesta de opinión, conformada por 7 preguntas, analizó el uso de canales de comunicación digitales de los estudiantes, la frecuencia de interacción, la red social más frecuentada, el acceso a publicaciones de ciencia y tecnología desde estas redes, el lenguaje utilizado y su conocimiento sobre el concepto de divulgación científica.

\subsection{Publicaciones sobre Ciencia y Tecnología en la Red Social Facebook}

En los resultados obtenidos de la encuesta de opinión se percibió un dato interesante, alrededor del $80 \%$ de los estudiantes encuestados de todas las universidades participantes respondieron que Facebook es la red social que más utilizan para informarse sobre los eventos que realizan en su universidad. Por lo que se consideró importante analizar las publicaciones de esta red social.

El análisis se realizó en los Fan pages de Facebook de las universidades seleccionadas correspondiente a febrero de 2016; se hace una síntesis de la información publicada en estos medios, y se clasifican las publicaciones de CyT para su análisis.

Como se lo mencionó previamente, el uso de estas dos herramientas de investigación permitirán analizar el manejo y uso de la red social Facebook por parte de las universidades, para realizar reflexiones sobre las prácticas más efectivas que permiten divulgar la cultura científica por estos medios. 


\section{4.- DISCUSIÓN Y RESULTADOS}

Como se lo ha explicado previamente, el estudio está compuesto de dos áreas de investigación, por un lado la encuesta aplicada a los estudiantes y en un segundo momento el análisis de las publicaciones de los fan pages de Facebook de las universidades participantes. A continuación se presentan los análisis de los resultados de estos procesos.

\subsection{Relación entre dinámica de los estudiantes y publicaciones de}

\section{Facebook}

\begin{tabular}{|c|c|c|c|c|c|c|}
\hline \multicolumn{7}{|c|}{ ¿Por qué canales de comunicación te enteras de los eventos que se } \\
realizan en tu universidad? \\
\hline \multicolumn{7}{|c|}{ Evaluación \% } \\
\hline Categoría & UCE & UDLA & PUCE & EPN & ESPE & USFQ \\
\hline Página Web & 6 & 4 & 6 & 34 & 18 & 14 \\
\hline $\begin{array}{c}\text { Redes } \\
\text { Sociales }\end{array}$ & 70 & 54 & 56 & 52 & 62 & 68 \\
\hline Carteleras & 10 & 2 & 4 & 0 & 0 & 6 \\
\hline $\begin{array}{c}\text { Pantallas } \\
\text { internas }\end{array}$ & 0 & 6 & 6 & 0 & 0 & 0 \\
\hline $\begin{array}{c}\text { Televisión } \\
\text { universitaria }\end{array}$ & 0 & 0 & 4 & 2 & 0 & 0 \\
\hline $\begin{array}{c}\text { Radio } \\
\text { Universitaria }\end{array}$ & 2 & 0 & 0 & 0 & 0 & 0 \\
\hline Amigos & 12 & 12 & 20 & 4 & 10 & 12 \\
\hline Otros & 0 & 22 & 4 & 8 & 10 & 0 \\
\hline
\end{tabular}

TABLA 3: Encuesta pregunta 1

Con porcentajes que varían entre el $52-70 \%$ los estudiantes encuestados manifiestan que utilizan mayoritariamente las redes sociales universitarias para enterarse de las noticias y los eventos que se realizan en su universidad. La incursión de las tecnologías de la información y comunicación han generado otras formas de interacción en las nuevas generaciones, por lo que este dato ratifica un modelo de consumo de medios digitales propio de este grupo personas.

Un dato interesante dentro de esta respuesta es que en la opción "otros", los estudiantes de 4 de las 6 universidades participantes (UDLA, PUCE, EPN y ESPE) manifiestan que también se enteran de los eventos de su universidad a través de las páginas de "Memes"; estas páginas son creadas por los estudiantes como un espacio de entretenimiento sobre situaciones en la universidad, pero demuestra que son consideradas una fuente de importante de información. Esta cifra permitiría reflexionar a las direcciones de comunicación de las universidades sobre el manejo actualizado de las plataformas digitales con publicaciones que causen interés en los estudiantes, y que sobretodo utilicen su lenguaje, para una mejor recepción y aceptación. 
Finalmente, otro dato importante es el que presenta la universidad de las Fuerzas Armadas (ESPE), que aun mantiene una estructura militar, algunas de las respuestas de los estudiantes indican que ellos se informan de las actividades que se realizan a través del minuto cívico ${ }^{4}$ los días lunes. En otras universidades como la UDLA se mantiene un fuerte el servicio de mailing como otra fuente de información, esto debido a que la universidad cuenta con plataformas de mail dirigidas a los estudiantes, mediante la que ellos se comunican constantemente con sus docentes. En la EPN, los usuarios manifiestan también en un alto porcentaje (32\%) que consultan la información a través de la página web, lo que demuestra que hay un notable interés en las publicaciones de la web de esta institución, por lo que el modelo utilizado está funcionando.

\begin{tabular}{|c|c|c|c|c|c|c|}
\hline \multicolumn{7}{|c|}{$\begin{array}{l}\text { ¿Con qué frecuencia visitas los medios digitales de tu universidad (página } \\
\text { web, facebook, twitter)? }\end{array}$} \\
\hline \multicolumn{7}{|c|}{ Evaluación \% } \\
\hline Categoría & UCE & UDLA & PUCE & EPN & ESPE & EPN \\
\hline Todos los días & 28 & 4 & 8 & 6 & 10 & 18 \\
\hline $\begin{array}{c}\text { Varias veces a } \\
\text { la semana }\end{array}$ & 24 & 14 & 22 & 10 & 18 & 28 \\
\hline $\begin{array}{c}\text { Dos veces a la } \\
\text { semana }\end{array}$ & 12 & 4 & 10 & 18 & 20 & 12 \\
\hline $\begin{array}{c}\text { Una vez a la } \\
\text { semana }\end{array}$ & 30 & 40 & 42 & 62 & 46 & 36 \\
\hline Nunca & 6 & 38 & 18 & 4 & 6 & 6 \\
\hline
\end{tabular}

TABLA 4: Encuesta pregunta 2

Con un porcentaje del $30-62 \%$ los estudiantes manifiestan que visitan los medios digitales universitarios una vez a la semana, lo cual indica un notorio interés por estar informados semanalmente de las actividades que se realizan en la universidad. Hay altos porcentajes como la UCE (28\%) en donde los estudiantes manifiestan que acceden a los medios digitales todos los días, en este caso sería importante conocer qué tipo de información revisan los estudiantes que tienen acceso constante a las plataformas digitales. Por otro lado, los estudiantes de la UDLA con un porcentaje elevado de respuestas (38\%) manifiestan que nunca ingresan a los medios digitales de la universidad, esta respuesta valida lo mencionado anteriormente, el uso de mailing en la UDLA fortalece la comunicación directa con los estudiantes por lo que no tienen un alto interés en el consumo de medios digitales propios de su institución.

\footnotetext{
${ }^{4}$ Minuto cívico es un espacio destinado para rendir honores a los símbolos patrios. Se realiza todos los días lunes, comúnmente en las escuelas y colegios, a primera hora de la mañana. Este espacio además se utiliza para informar a los estudiantes sobre actividades que se llevarán a cabo durante toda la semana en la institución.
} 


\begin{tabular}{|c|c|c|c|c|c|c|}
\hline \multicolumn{7}{|c|}{ EVuál es la red social universitaria que más visitas? } \\
\hline Categoría & UCE & UDLA & PUCE & EPN & ESPE & USFQ \\
\hline Facebook & 89,80 & 78 & 90 & 93,62 & 93,18 & 84 \\
\hline Twitter & 2,04 & 16 & 2 & 4,26 & 4,55 & 0 \\
\hline Instagram & 0 & 4 & 4 & 0 & 0 & 8 \\
\hline Otros & 8,16 & 12 & 4 & 2,13 & 2,27 & 8 \\
\hline
\end{tabular}

TABLA 5: Encuesta pregunta 3

Claramente se demuestra que la red social más visitada por los estudiantes universitarios es Facebook. Esta respuesta es validada de acuerdo a una investigación presentada por Cobertura digital (2015) en la que presenta el fuerte crecimiento de Facebook en los últimos seis años. En Ecuador esta red social creció de 400.000 usuarios en el 2009 a 8 millones de usuarios en el 2015. También el Instituto de Estadísticas y Censos (INEC) $)^{5}$ realizó una encuesta en 2014 para analizar las preferencias por redes sociales en el país y los datos presentan que un $41,4 \%$ de personas encuestadas tienen cuentas de redes sociales activas y Facebook tiene ampliamente la aceptación de los usuarios.

Este dato es muy importante dentro de esta investigación puesto que al ser facebook la principal fuente de información por parte de los estudiantes, se entiende que se encuentra actualizada constantemente por los encargados de comunicación de las universidades. En el caso de Twitter, aunque reducido, se presenta un resultado $(2,04-4,55 \%)$ que demuestra que hay un porcentaje de estudiantes que siguen estas cuentas. En lo que se refiere a Instagram hay un porcentaje presentado (4$8 \%$ ), dato que demuestra el interés de por seguir una red social que publica fotos de la cotidianidad universitaria. Finalmente en Otros, hay estudiantes que vuelven a mencionar las páginas de MEMES antes explicadas.

\begin{tabular}{|c|c|c|c|c|c|c|}
\hline \multicolumn{7}{|c|}{ ¿Has encontrado información sobre investigación, ciencia y tecnología en } \\
las redes sociales de tu universidad? \\
\hline Cvaluación \% \\
\hline Categoría & UCE & UDLA & PUCE & EPN & ESPE & USFQ \\
\hline SI & 72 & 36 & 50 & 93,62 & 77,55 & 84 \\
\hline NO & 28 & 64 & 50 & 6,38 & 22,45 & 16 \\
\hline
\end{tabular}

TABLA 6: Encuesta pregunta 4

\footnotetext{
${ }^{5}$ Acceso a INEC Ecuador http://www.ecuadorencifras.gob.ec
} 
Las respuestas a esta pregunta indican que hay opiniones divididas en torno a la información de ciencia y tecnología publicada en redes sociales. Los datos que más sorprenden son los de la UDLA (64\%) en donde los encuestados indican en una amplia mayoría que no hay información de CyT en las redes universitarias; Aquí se debería analizar qué tipo de publicaciones predominan en redes sociales para que exista esta respuesta por parte de los estudiantes. En el caso de la PUCE (50\%) la respuesta es interesante, porque los estudiantes indican en porcentajes similares que encuentran y no encuentran este tipo de información en las redes sociales.

\begin{tabular}{|l|c|c|c|c|c|c|}
\hline \multicolumn{7}{|c|}{ ¿Qué tipo de información científica has encontrado en las redes sociales de } \\
tu universidad? \\
\hline \multicolumn{7}{|c|}{ Evaluación \% } \\
\hline \multicolumn{1}{|c|}{ Categoría } & UCE & UDLA & PUCE & EPN & ESPE & USFQ \\
\hline $\begin{array}{l}\text { Estudios o investigaciones } \\
\text { realizadas en tu } \\
\text { universidad }\end{array}$ & 35,56 & 26,19 & 36,36 & 51,02 & 43,18 & 48,98 \\
\hline $\begin{array}{l}\text { Descubrimientos } \\
\text { científicos }\end{array}$ & 4,44 & 9,52 & 4,55 & 4,08 & 0 & 4,08 \\
\hline $\begin{array}{l}\text { Entrevistas a } \\
\text { investigadores }\end{array}$ & 4,44 & 14,29 & 15,91 & 10,20 & 20,45 & 8,16 \\
\hline $\begin{array}{l}\text { Proyectos de innovación } \\
\text { universitarios }\end{array}$ & 46,67 & 45,24 & 27,27 & 26,53 & 36,36 & 28,57 \\
\hline $\begin{array}{l}\text { Estudios e investigaciones } \\
\text { extranjeras }\end{array}$ & 8,89 & 4,76 & 15,91 & 8,16 & 0 & 8,16 \\
\hline $\begin{array}{l}\text { Otros } \\
\text { TABLA 7: Encuesta pregunta }\end{array}$ & 0 & 0 & 0 & 0 & 0 & 2,04 \\
\hline
\end{tabular}

TABLA 7: Encuesta pregunta 5

Los datos de estas respuestas también son interesantes. En la UCE $(46,67 \%)$ y UDLA $(45,24)$ indican que la información que más se destaca en las redes digitales universitarias está relacionada con proyectos de innovación universitarios, esta respuesta indica que se publica constantemente los novedosos resultados de proyectos realizados por los estudiantes. En la PUCE (36,36\%), ESPE $(43,18 \%)$, EPN $(51,02 \%)$ y USFQ $(48,98 \%)$ se destaca la publicación de estudios o investigaciones realizadas en las universidades, lo que demuestra una divulgación no solo de proyectos de innovación como en el caso de otras instituciones, sino también de otro tipo de estudios que no únicamente están relacionados con proyectos prácticos, y se relacionan a la ciencia en general. Este dato puede demostrar en cierta medida qué tipo de publicaciones se encuentran.

\begin{tabular}{|l|r|r|r|r|r|r|}
\hline \multicolumn{7}{|c|}{ ¿Qué tipo de lenguaje utilizan estas publicaciones? } \\
\hline Categoría & UCE & UDLA & PUCE & EPN & ESPE & USFQ \\
\hline Claro & 24 & 8,33 & 28,57 & 8 & 10,87 & 32 \\
\hline Comprensible & 44 & 33,33 & 36,73 & 82 & 60,87 & 38 \\
\hline
\end{tabular}




\begin{tabular}{|l|r|r|r|r|r|r|} 
Amigable & 14 & 18,75 & 14,29 & 8 & 17,39 & 12 \\
\hline Muy Técnico & 12 & 29,17 & 14,29 & 2 & 6,52 & 14 \\
\hline Confuso & 2 & 6,25 & 4,08 & 0 & 4,35 & 2 \\
\hline Otros & 4 & 4,17 & 2,04 & 0 & 0 & 2 \\
\hline
\end{tabular}

TABLA 8: Encuesta pregunta 6

De acuerdo a los datos proporcionados en esta pregunta, la mayoría de los encuestados consideran que el lenguaje utilizado en las publicaciones realizadas en las redes sociales universitarias es claro, comprensible y amigable, lo que demuestra que los visitantes de estas redes sociales comprenden claramente los conceptos científicos y técnicos expresados en las publicación, y además éstas intentan manejar términos de fácil interpretación para el público al que se dirigen.

Sin embargo, en cuatro de las seis universidades estudiadas se encuentra un alto porcentaje que manifiesta que el lenguaje utilizado en las publicaciones es muy técnico; así lo reflejan los datos: UCE $(12 \%)$, UDLA $(29,17 \%)$, PUCE $(14,29)$ Y USFQ (14\%). Estos datos permiten reflexionar que quizá no todas las publicaciones de facebook se encuentra en lenguajes sencillos y de fácil compresión por parte de los estudiantes.

\begin{tabular}{|l|c|c|c|c|c|c|c|}
\hline \multicolumn{7}{|c|}{ ¿Qué es para ti divulgación de la ciencia? } \\
\hline \multicolumn{1}{|c|}{ Categoría } & UCE & UDLA & PUCE & EPN & ESPE & USFQ \\
\hline $\begin{array}{l}\text { Compartir información científica } \\
\text { entre pares expertos }\end{array}$ & 6 & 2 & 6 & 4 & 2 & 4 \\
\hline $\begin{array}{l}\text { Compartir información científica } \\
\text { de forma abierta y pública con } \\
\text { diferentes actores, no } \\
\text { necesariamente científicos. }\end{array}$ & 82 & 78 & 66 & 80 & 74 & 64 \\
$\begin{array}{l}\text { Compartir información científica } \\
\text { en medios masivos de } \\
\text { comunicación (televisión, radio, } \\
\text { prensa, internet) }\end{array}$ & 12 & 20 & 28 & 16 & 24 & 32 \\
\hline
\end{tabular}

TABLA 9: Encuesta pregunta 7

En esta pregunta se evidencia claramente la amplia comprensión que tienen los estudiantes sobre el significado de divulgación de la ciencia, desde el $65 \%$ (USFQ) al $82 \%$ (UCE), sobre el concepto de divulgación científica "Compartir información científica de forma abierta y pública con diferentes actores, no necesariamente científicos".

Sin embargo, también se destaca en un porcentaje, no tan elevado, del $12 \%$ (UCE) al 32\% (USFQ), la comprensión del concepto de divulgación científica como: "Compartir información científica en medios masivos de comunicación (televisión, radio, prensa, internet)", concepto que está vinculado a la comunicación mediática, que tiene relación a las respuestas de los estudiantes debido a su acercamiento a la 
ciencia mediante programas de televisión y revistas especializadas en el tema, más no a otras actividades que se realicen públicamente.

\subsection{Publicaciones de Facebook}

Luego del análisis de resultados de la encuesta a los estudiantes, a continuación se presenta una contextualización de las publicaciones generadas en los fan pages de Facebook de las universidades de febrero 2016, con el número de publicaciones de ciencia y tecnología generadas.

\begin{tabular}{|c|c|c|c|}
\hline \multirow{2}{*}{ TABLA 9 } & \multicolumn{3}{|c|}{$\begin{array}{c}\text { PUBLICACIONES EN FACEBOOK } \\
\text { FEBRERO 2016 }\end{array}$} \\
\hline Universidad & Seguidores & \# Publicaciones & $\begin{array}{c}\text { \# Publicaciones } \\
\text { CyT }\end{array}$ \\
\hline USFQ & 819.474 & 57 & 1 \\
\hline UDLA & 479.391 & 74 & 6 \\
\hline UCE & 53.551 & 58 & 9 \\
\hline PUCE & 50.302 & 23 & 1 \\
\hline ESPE & 45.698 & 31 & 2 \\
\hline EPN & 24.021 & 38 & 17 \\
\hline
\end{tabular}

TABLA 10: Publicaciones de Facebook Universidades

En el cuadro presentado se ha incluido el número de seguidores de cada uno de los fan pages, las publicaciones del mes y las que están relacionadas con ciencia y tecnología.

La USFQ ${ }^{6}$ es la universidad que más seguidores presenta (819.474), tomando en consideración que el número de estudiantes de esta institución actualmente corresponde a 6.000 , esta cuenta tiene como seguidores a públicos internos y externos de la universidad. En el mes de febrero realizó 57 publicaciones en su fan page y sólo 1 de estas publicaciones corresponde a CyT. La publicación hace referencia a la invitación de una conferencia sobre "Ecología y Evolución de las Enfermedades Transmisibles", no genera mayor información al respecto y solo se lo puede considerar como una publicación de información.

Lo que se percibe dentro de la página web es que este fan page tiene acceso a otros micrositios de la universidad y otras cuentas de facebook de cada uno de los colegios (facultades) de la universidad en donde se publica información específica, pero no se publica en la página oficial de facebook. Sería importante para los encargados de comunicación de esta institución fortalecer las publicaciones de ciencia y tecnología

\footnotetext{
${ }^{6}$ Acceso Fan page USFQ https://goo.gl/NIngBd
} 
en este espacio, cuando cuentan con un amplio porcentaje de público perceptor para fortalecer la cultura científica.

La UDLA ${ }^{7}$ es la segunda universidad con mayor número de seguidores (479.391) en su fan page oficial. En el mes de febrero tienen 74 publicaciones, y 6 relacionadas con CyT. En 2 de las 6 publicaciones mencionan a los artículos de opinión del investigador César Paz y Miño y las otras 4 hablan sobre investigaciones realizadas sobre el virus Zika que actualmente es de interés nacional. Por lo que se puede visualizar con estos datos, esta institución realiza divulgación científica de las investigaciones que genera, además de promocionar otros productos comunicacionales realizados por sus investigadores.

En cuanto se refiere a la UCE $^{8}$ con un total de 53.551 seguidores, en el mes de febrero se encuentran 58 publicaciones y 9 están relacionadas a CyT. Estas publicaciones hacen referencias a actividades realizadas por los estudiantes en cuanto a cursos de especialización, casas abiertas, conferencias, difusión de notas de prensa sobre investigaciones realizadas que han sido publicadas en medios impresos. La institución publica constantemente información sobre los estudiantes, al ser ellos el principal público perceptor en esta plataforma digital.

La PUCE ${ }^{9}$ con 50.302 de seguidores es la institución que menos publicaciones tiene en esta red social, se cuentan 23 en el mes de febrero y 1 sobre CyT en donde se invita a los estudiantes a registrarse a un evento específico sobre "Investigación en enfermedades tropicales", no se refleja mayor movimiento en esta red social y en la página de facebook no se encuentran micrositios de acceso a otras páginas donde se pueda conseguir mayor información. Sería importante para la institución fortalecer este canal cono estrategias comunicacionales que permitan conocer el accionar investigativo de sus estudiantes, docentes y de toda la comunidad universitaria.

Por otro lado la ESPE ${ }^{10}$ con 45.698 seguidores tiene 31 publicaciones en el mes de febrero y solo 2 sobre CyT. Las publicaciones hacen referencia sobre un curso de Transferencia tecnológica y el otro sobre la firma de un convenio con el Consejo de Gobierno de Galápagos y la Fundación Charles Darwin. Aunque en la página web no se realiza constante divulgación científica de las actividades que se generan, la ESPE cuenta con otro fan page denominado "E-Ciencia" en donde realizan estrategias de divulgación con sus diferentes públicos, sin embargo, esta cuenta con es completamente reconocida por todos los estudiantes de la universidad. Sería importante que se pueda coordinar mejor las publicaciones que se unifiquen en un solo espacio de mayor acceso para la comunidad universitaria.

\footnotetext{
${ }^{7}$ Acceso fan page UDLA https://goo.gl/VRn3c]

${ }^{8}$ Acceso fan page UCE https://goo.gl/yhSTsR

${ }^{9}$ Acceso fan page PUCE https://goo.gl/gNp4ac

${ }^{10}$ Acceso fan page ESPE https://goo.gl/TxTGO2
} 
Finalmente la EPN ${ }^{11}$ es la última institución en este cuadro, con 24.021 seguidores, tiene 38 publicaciones en febrero, sin embargo 17 son de CyT, de acuerdo a los datos es la institución que más publicaciones tiene en este campo con relación a las demás investigadas. Sus publicaciones están relacionadas a noticias de CyT de agencias internacionales, invitación a eventos y entrevistas a investigadores. Aunque es el fan page que menos seguidores tiene es el que más información sobre CyT, información variada que como se menciona anteriormente incluye noticias y datos interesantes sobre el tema. En este caso se nota el interés que tiene la institución por fortalecer este canal de comunicación como espacio de divulgación con los estudiantes.

A continuación se presenta un dato interesante sobre las publicaciones científicas realizadas por las universidades de Ecuador de 2014-2015. Esta es un ranking presentado por SCImago Research Groupen donde se detallan las instituciones de educación superior ecuatorianas con el número de publicaciones en SCOPUS, Colaboración Internacional, Publicaciones de Alta Calidad y Porcentaje de Excelencia.

\begin{tabular}{|c|c|c|c|c|c|}
\hline POSICIÓN & NOMBRE & $\begin{array}{l}\text { \# Publicaciones } \\
\text { Científicas } \\
\text { SCOPUS }\end{array}$ & $\begin{array}{l}\text { \% Publicaciones } \\
\text { Colaboración } \\
\text { Internacional }\end{array}$ & $\begin{array}{l}\text { \% Publicaciones } \\
\text { Alta Calidad }\end{array}$ & $\begin{array}{c}\% \\
\text { Excelencia }\end{array}$ \\
\hline 1 & $\begin{array}{l}\text { Universidad } \\
\text { San Francisco } \\
\text { de Quito USFQ }\end{array}$ & 422 & 92,89 & 74,17 & 26,81 \\
\hline 2 & $\begin{array}{c}\text { Pontificia } \\
\text { Universidad } \\
\text { Católica del } \\
\text { Ecuador PUCE }\end{array}$ & 319 & 81,19 & 53,61 & 12,34 \\
\hline 3 & $\begin{array}{c}\text { Escuela } \\
\text { Politécnica } \\
\text { Nacional EPN }\end{array}$ & 152 & 85,53 & 61,18 & 6,21 \\
\hline 4 & $\begin{array}{l}\text { Universidad } \\
\text { Técnica } \\
\text { Particular de } \\
\text { Loja UTPL }\end{array}$ & 143 & 69,23 & 31,47 & 6,47 \\
\hline 5 & $\begin{array}{c}\text { Escuela } \\
\text { Superior } \\
\text { Politécnica del } \\
\text { Litoral }\end{array}$ & 137 & 79,64 & 32,85 & 13,08 \\
\hline 6 & $\begin{array}{l}\text { Universidad } \\
\text { Central del } \\
\text { Ecuador UCE }\end{array}$ & 103 & 70,87 & 40,78 & 3,92 \\
\hline 7 & $\begin{array}{c}\text { Universidad de } \\
\text { Cuenca }\end{array}$ & 91 & 89,01 & 42,86 & 14,44 \\
\hline 8 & $\begin{array}{l}\text { Universidad } \\
\text { Católica de } \\
\text { Santiago de } \\
\text { Guayaquil }\end{array}$ & 90 & 73,33 & 33,33 & 20,22 \\
\hline
\end{tabular}

\footnotetext{
${ }^{11}$ Acceso fan page EPN https://goo.gl/kvVAbw
} 


\begin{tabular}{|c|c|c|c|c|c|}
\hline 9 & $\begin{array}{c}\text { Universidad de } \\
\text { las Fuerzas } \\
\text { Armadas ESPE }\end{array}$ & 53 & 79,25 & 37,74 & 8,7 \\
\hline 10 & $\begin{array}{c}\text { Universidad } \\
\text { Tecnológica } \\
\text { Indoamérica }\end{array}$ & 42 & 78,57 & 42,86 & 12,2 \\
\hline 11 & $\begin{array}{c}\text { Universidad de } \\
\text { Especialidades } \\
\text { Espíritu Santo }\end{array}$ & 34 & 35,29 & 26,47 & 5,88 \\
\hline 11 & $\begin{array}{c}\text { Universidad de } \\
\text { Guayaquil }\end{array}$ & 34 & 91,18 & 44,12 & 3,03 \\
\hline 12 & $\begin{array}{c}\text { Universidad } \\
\text { Politécnica } \\
\text { Salesiana }\end{array}$ & 32 & 59,38 & 6,25 & 0 \\
\hline 13 & $\begin{array}{c}\text { Universidad } \\
\text { Nacional de } \\
\text { Loja }\end{array}$ & 29 & 93,1 & 48,28 & 6,9 \\
\hline 15 & $\begin{array}{c}\text { Universidad de } \\
\text { Las Américas } \\
\text { UDLA }\end{array}$ & 25 & 44 & 32 & 4 \\
\hline $\begin{array}{c}\text { Universidad } \\
\text { Andina } \\
\text { SimónBolívar }\end{array}$ & 22 & 54,55 & 9 & \\
\hline
\end{tabular}

Tabla 2: Ranking y Producción científica de universidades en Ecuador.

Fuente: SCImago Research Group, 2015

De acuerdo a la información presentada, las 6 universidades de la muestra se encuentran en el ranking de las 15 universidades en Ecuador con mayor producción científica. La institución que lidera el estudio es la USFQ (422 publicaciones), que de acuerdo al análisis presentado en este artículo es una de las universidades que más realiza divulgación científica desde diferentes cuentas de sus colegios (facultades); sin embargo no se potencia en el fan page oficial de la universidad.

En segundo lugar se encuentra la PUCE (319 publicaciones), sin embargo denota claramente que no realizan divulgación de la producción científica que generan, al menos no lo realizan en los medios digitales de la universidad, por lo que podría analizarse que otras estrategias de comunicación pública utilizan. Sería importante para la universidad utilizar la red social de facebook y presentar publicaciones cortas y sencillas sobre las investigaciones que realizan, sino se sigue manteniendo un único modelo de difusión especializada entre expertos.

En tercer lugar del Ranking se encuentra la EPN (152 publicaciones), que de acuerdo a los análisis anteriores se denota su interés no solo en la producción científica sino también a la divulgación de la ciencia. Aunque la mayoría de publicaciones de la EPN son relacionadas a charlas sobre CyT, se podría posteriormente hacer un análisis sobre si son los investigadores que dan las charlas los mismos que generan la producción científica en la universidad. Aquí la universidad podría ampliar las estrategias de divulgación en su facebook, incluyendo más entrevistas y videos dinámicos que expliquen las investigaciones realizadas lo que fortalecería el interés de los públicos que les siguen en esta red social. 
La UCE aparece en un 60lugar dentro de la tabla con (103 publicaciones), aunque de acuerdo al análisis de este artículo es la segunda que presenta más publicaciones sobre CyT en facebook, podría mejorar su divulgación incluyendo estrategias de comunicación que incluyan vocerías con los investigadores de la universidad que contextualicen los proyectos realizados.

En 9no lugar se encuentra la ESPE con (53 publicaciones), una cifra baja de acuerdo al ranking, pero como se indicó en este análisis, ellos realizan divulgación de las actividades que generan, no por el fan page oficial, pero si por otros medios.

Finalmente se encuentra la UDLA (25 publicaciones), la institución que menos publicación científica realiza de acuerdo al ranking, pero de acuerdo a este análisis es la que más promociona a los investigadores de su universidad y los procesos de comunicación que realizan.

\section{5.- CONCLUSIONES}

Una vez realizado este análisis sobre la interacción de los estudiantes con los medios digitales de sus universidades y la dinámica de Facebook, se concluye que las universidades ecuatorianas de la muestra realizan poca divulgación científica en general y también en los fan pages oficiales de Facebook sobre la producción científica que generan. El interés está en publicar más información relacionada con temas generales de la universidad tales como invitación a cursos, seminarios, información administrativa, eventos deportivos, noticias sobre la actividad del rector y otras autoridades, etc.

Por otro lado, en el tema de divulgación de CyT públicamente no se encuentra definida una estrategia sobre qué publicar, cómo y dónde hacerlo. Quizás solo destaque el caso de la EPN, que es la que más publicaciones tiene en el mes estudiado, y se categoriza publicaciones sobre entrevistas a sus investigadores y notas de prensa sobre los proyectos que están realizando. Una mejor codificación de las actividades de CyT para publicar en fan pages podría optimizar las estrategias de divulgación de ciencia de las universidades. Esta codificación puede hacerse pensando en la diversidad de eventos: casas abiertas, seminarios, foros, cafés científicos y otros productos comunicacionales como: entrevistas, presencia en medios, micro notas y videos sobre investigaciones, etc.

Algunas universidades cuentan con otro tipo fan pages para divulgar sus actividades, sin embargo sería importante que se pueda potenciar el fan page oficial de la universidad para que sea considerado como una principal fuente de información no solo de los temas de acontecer universitarios, sino de temas como los de CyT que potencian el desarrollo de cultura científica en la comunidad universitaria. Se considera un buen ejemplo de esto lo que hace la EPN, que además de publicar 
información de sus investigadores, coloca noticias y datos interesantes sobre CyT, lo que genera interés en sus seguidores. Sin embargo, en el caso de esta institución, deberían pensar en una nueva estrategia para ampliar su número de seguidores.

Esta conclusión es similar a la que proponen Brito, J. G. Laaser, W. Toloza, E. A. (2012) en su estudio El uso de redes sociales por parte de las universidades a nivel institucional, en la que manifiestan que es necesario para las universidades mantener un único perfil institucional centralizado. Como es de suponer, este modelo es complejo de aplicarlo en universidades medianas o grandes, en las que resulta prácticamente imposible homogeneizar y canalizar la información de los institutos/facultades descentralizadas. Asimismo, el posible control central resulta contradictorio con la filosofía de libertad que encarnan las redes, sin embargo es una acción que mejora el perfil institucional de las universidades.

La divulgación necesita de una actitud proactiva para despertar el interés social por la ciencia. Se debe involucrar activamente a los investigadores en las planificación y ejecución de actividades de divulgación universitaria, ya que si su actitud es negativa o de rechazo, choca con la poca valoración que pueda tener sobre esta necesidad y la ventaja que supone el reconocimiento social de su labor. La divulgación debería llegar a un importante segmento de la población universitaria y no universitaria, no solo difundiéndola entre los que ya muestran interés, sino también ganando adeptos, ya que la demanda nacerá del conocimiento (Lascurain, M. y Sanz, Elías, 2009).

Es importante también incluir estrategias novedosas para los estudiantes. El estudio ya muestra que una principal fuente de información son los fan pages de "Memes" de las universidades, por su constante actualización, dinámica y uso de recursos y lenguajes que generan expectativa e interés en los jóvenes. Las redes sociales son plataformas informales de interacción continua, por lo que los encargados de manejarlas pueden utilizar recursos atractivos, desde los colores hasta productos comunicacionales para captar a sus perceptores, es decir desarrollando una gestión de contenidos multimedia en estas plataformas.

En este sentido, es importante que las universidades consideren la necesidad de crear departamentos de comunicación especializados sobre comunicación científica, o bien formar a personal específicamente en tareas de divulgación de la CyT, en donde se promuevan actitudes para divulgar. La divulgación de la ciencia no puede ser considerada una actividad intuitiva ni voluntaria para los investigadores, al contrario debe incluirse dentro de una planificación institucionalizada y sistemática que requiere contenidos curriculares en la que se unifiquen conocimientos procedentes de la realidad científica con técnicas y metodologías implicadas en la comunicación como parte de la formación científica (Lascurain, M. y Sanz, Elías, 2009).

Finalmente este primer acercamiento a las universidades ecuatorianas muestra que no hay estrategias definidas en cuanto a la divulgación científica, que las pocas acciones son aleatorias y coyunturales y se llevan a cabo de acuerdo a temas de 
interés nacional o universitario sobre CyT. Sin embargo no se encuentra una estrategia específica para divulgar información de CyT que fortalezca la construcción de una cultura científica, sobretodo en el caso de facebook, teniendo en cuenta su potencialidad como principal fuente de información para los estudiantes de acuerdo a los datos expuestos. Si en las universidades ecuatorianas se incluyen cursos sobre difusión para la redacción de artículos científicos tanto en pregrado como en posgrado, también es necesario incluir capacitaciones en divulgación de la ciencia, en donde tanto estudiantes como docentes puedan aprender de esta mediación comunicativa. La divulgación está basada en la mediación ya que, si la comunicación entre el especialista y el público en general es complicada, se requiere un tercer actor (el mediador) que traduzca el lenguaje del investigador para suscitar interés, curiosidad y emoción Calvo (2000). Este estudio preliminar permite por tanto definir futuras líneas de investigación de la tesis doctoral, como es el caso de una categorización de actividades sobre CyT que realizan las universidades seleccionadas, abordar su dinámica y medir su impacto, para armar una propuesta de plan con las buenas prácticas encontradas en cada institución sobre la forma más efectiva para divulgar ciencia en la universidad ecuatoriana.

\section{6.- REFERENCIAS}

Brito, J. G. Laaser, W. Toloza, E. A. (2012) El uso de redes sociales por parte de las universidades a nivel institucional. Un estudio comparativo. RED, Revista de Educación a Distancia. Número 32. Recuperado en: http://www.um.es/ead/red/32. Consultado el 23/05/2016.

Calvo Hernando, Manuel. (2000). El Nuevo Periodismo de la Ciencia. Quito: Ediciones Ciespal.

Cobertura Digital (2015) Redes sociales Ecuador: Facebook pasó los 8 millones 2015. Recuperado en: http://goo.gl/zGzkf] Consultado el: 10/03/2016.

Erazo, Ma. de los Ángeles. (2007)Comunicación, Divulgación y Periodismo de la Ciencia, Una necesidad imprescindible para Ibero América. Quito: Editorial Planeta.

Fayard, Pierre. (2004).La Comunicación Pública de la Ciencia. México D.F.: UNAMDirección General de Divulgación de la Ciencia.

Lascurain, M. \& Sanz, E. (2009).La divulgación científica en el entorno universitario.Coimbra: Universidad de Coimbra.

Lozano, M. (2003). La investigación como estrategia para la apropiación social de la ciencia y la tecnología". Había una vez una iguana: Experiencias en apropiación social de la ciencia y la tecnología. Bogotá: Gobierno del Departamento de Guajira. 
Salazar, Andrea (2015). La construcción de las políticas públicas de ciencia, tecnología e innovación en la República del Ecuador (Período 2007-2015). Tesis inédita de maestría. Universidad de Buenos Aires, Buenos Aires.

Scimago Research Group (2015). Ranking y producción científica de universidades en Ecuador. Recuperado en: http://www.scimagoir.com. Consultado el: 15/03/2016.

Vaccarezza, L. S. (2008). Exploraciones en torno al concepto de cultura científica.En FECYT, Resúmenes del Congreso Iberoamericano de Ciudadanía y PolíticasPúblicas de Ciencia y Tecnología. Madrid. 